\title{
Alguns teoremas clássicos da geometria sintética e aplicações
}

\author{
Some Classical Theorems of Synthetic Geometry and Applications
}

\author{
Vinícius Paulo Freitas ${ }^{1}$ e Nilomar Vieira Oliveira ${ }^{2}$ \\ ${ }^{1}$ Docente - Instituto Federal de Educação, Ciência e Tecnologia do Amazonas, AM - Brasil \\ viniciusmat2003@yahoo.com.br \\ ${ }^{2}$ Docente - Universidade Federal do Amazona, AM - Brasil \\ nilomar@gmail.com
}

\begin{abstract}
Resumo
O presente artigo é fruto de uma parte da dissertação de mestrado, onde procuramos fazer uma abordagem simples de alguns teoremas clássicos da Geometria Euclidiana Plana e torná-los mais conhecidos, pois embora tenham um grande papel na resolução de muitos problemas geométricos, estão de certa forma esquecidos tanto no ensino básico quanto no ensino de graduação. No intuito de resgatar tais teoremas, desenvolvendo assim habilidades em Geometria, exploramos os seguintes teoremas: Menelaus, Ceva, Ptolomeu, Hiparco,Desargues, Feuerbach. Para as demonstrações destes teoremas, fizemos o uso de alguns resultados da Geometria Plana e da Geometria Inversiva. Acreditamos que tanto o enfoque da realização desse trabalho, com a utilização da Geometria Inversiva, por exemplo, como os teoremas clássicos, que utilizamos simplesmente métodos elementares da Geometria Sintética, pode servir para a melhoria do ensino-aprendizagem de Geometria Euclidiana Plana e possivelmente servir de elemento motivador para alunos e professores que busquem aprimorar seus conhecimentos em Geometria nos seus diversos desdobramentos.

Palavras-chave: Ensino-Aprendizagem, Geometria Euclidiana, Teoremas Clássicos.
\end{abstract}

\begin{abstract}
This article is based on a part of the dissertation, where we try a simple approach of some classical theorems of Euclidean geometry Plana and make them better known, because although they have a great role in solving many geometrical problems, they are somehow forgotten both in primary and in undergraduate education. In order to redeem such theorems, therefore developing the people skills in Geometry, we explored the following Theorems:Menelaus, Ceva, Ptolemy, Hipparchus, Desargues, and Feuerbach. For the proofs of the mentioned theorems, we use some results of Plane Geometry and Inversive Geometry. We believe that both approach the making of this work, with the use of Inversive Geometry, for instance, as the classical theorems which we used only elementary methods of Synthetic Geometry, can serve to improve the teaching and learning of Euclidean Plane Geometry and possibly serve as the motivating element for students and teachers seeking to improve their knowledge in Geometry in its various ramifications.
\end{abstract}

Keywords: Teaching-Learning, Euclidean Geometry, Classical Theorems. 


\section{Introdução}

Atualmente com a decadência do ensino de Geometria na educação básica brasileira, tem-se aumentado o interesse de pesquisadores de Ensino da Matemática em debater esta problemática nas escolas e, até mesmo, nas Universidades. "Além disso, há uma consciência coletiva da importância deste ramo da Matemática, como área de aprendizagem, pela sua contribuição na formação e cultura dos alunos e pela aplicação que tem em outras Ciências, inclusive dentro da própria Matemática" (SARMENTO, 2007).

Foi nesse intuito de tornar o ensino de Geometria mais interessante e resgatar alguns de seus resultados clássicos já até esquecidos no ensino básico e, geralmente, no ensino de graduação, é que foi desenvolvido este trabalho, objetivando criar um roteiro de estudo direcionado aos níveis de educação supracitados. A ideia é desenvolver habilidades em Geometria, explorando "alguns teoremas clássicos da Geometria Euclidiana", tais como os Teoremas de Menelaus e Ceva, que tratam, respectivamente, de problemas de colinearidades e concorrências; o Teorema de Ptolomeu, que relaciona as diagonais e os lados de um quadrilátero inscritível, e também nos dá uma condição de inscrição de quadriláteros sem a utilização de análise ângulos; a Circunferência dos nove pontos, que tratam de resultados surpreendentes envolvendo alguns pontos notáveis dos triângulos; o Teorema de Feuerbach, que fornece uma propriedade importante da Circunferência dos nove pontos, e outros não menos interessantes que serão chamados, neste trabalho, de "aplicações", pois seguem diretamente dos teoremas acima citados.

\section{Os Teoremas Clássicos Abordados}

\subsection{O Teorema de Menelaus e o Teorema de Ceva}

Muitos dos problemas envolvendo triângulos estão relacionados com conjuntos de determinados pontos que são colineares, ou com conjuntos de segmentos que são concorrentes. Problemas estes que podem ser solucionados através de dois Teoremas Clássicos da Geometria Euclidiana: O Teorema de Menelaus e o Teorema de Ceva. O primeiro trata de colinearidade de pontos e o segundo de concorrência de segmentos. Com a ajuda destes dois teoremas, provas que envolvem colinearidade e concorrência, que antes eram longas e complicadas, passaram a ser simplificada, tornando-as acessíveis a todos os níveis de ensino.

Como o Teorema de Menelaus faz uso de razão orientada de segmentos colineares, faremos um breve resumo sobre este assunto antes de sua demonstração.
Definição 2.1. (Razão orientada) $A$ razão orientada $r=$ $(M, \overleftrightarrow{A B})$, em que o ponto $M$ divide a reta $\overleftrightarrow{A B}$, é um número tal que $|r|=\frac{A M}{M B}$.

Dada esta definição, a pergunta agora é: Como sabermos se o ponto $M$ está ou não entre os pontos $A$ e $B$ ? A resposta vem das propriedades da razão orientada de segmentos, que são elas:

- Se $M \in \overline{A B}$, então $\frac{A M}{M B}>0$. De fato, neste caso $\overrightarrow{A B}$ e $\overrightarrow{M B}$ tem a mesma orientação.

- Se $M \notin \overline{A B}$, então $\frac{A M}{M B}<0$. De fato, neste caso $\overrightarrow{A M}$ e $\overrightarrow{M B}$ tem orientação oposta.

Para o Teorema de Menelaus, consideraremos apenas as medidas que levam em conta a orientação dos segmentos, que são chamadas de medidas algébricas. Já aquelas medidas que não levam em consideração a orientação do segmento são chamadas de medidas geométricas.

Teorema 2.1. (Teorema de Menelaus) Sejam três pontos $L, M$ e $N$ localizados respectivamente nas retas suportes dos lados $\overline{A B}, \overline{B C}$ e $\overline{C A}$ de um triângulo $A B C$ (qualquer) e diferentes dos vértices. Então L, Me $N$ são colineares se, e somente se

$$
\frac{L A}{L B} \cdot \frac{M B}{M C} \cdot \frac{N C}{N A}=1
$$

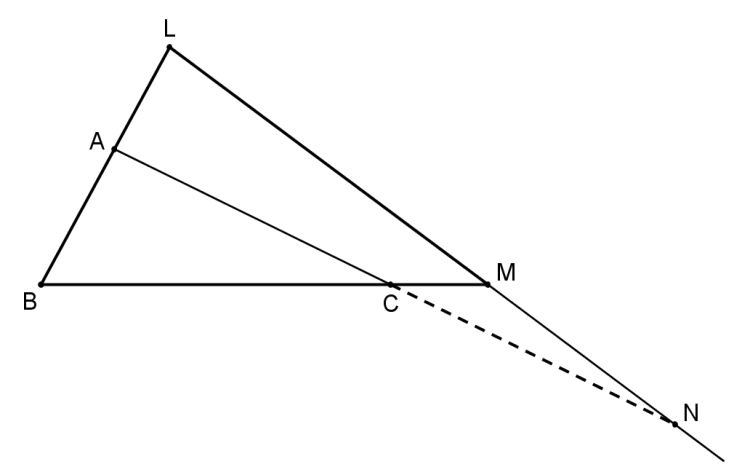

Figura 1: Teorema de Menelaus.

Demonstração. Seja o $\triangle A B C$, e sejam $L, M$ e $N$ pontos colineares pertencentes as retas $\overleftrightarrow{A B}, \overleftrightarrow{B C}$ e $\overleftrightarrow{C A}$, respectivamente. Pelo vértice $\mathrm{A}$, traça-se uma reta $\overleftrightarrow{A D}$ paralela a transversal $\overleftrightarrow{L M}$.

Pelo Teorema de Tales as paralelas $\overleftrightarrow{A D}$ e $\overleftrightarrow{L M}$ cortam as secantes $\overleftrightarrow{A B}$ e $\overleftrightarrow{B C}$ em partes proporcionais, daí

$$
\frac{L A}{M D}=\frac{L B}{M B} \Longrightarrow \frac{L A}{M D} \cdot \frac{M B}{L B}=1
$$




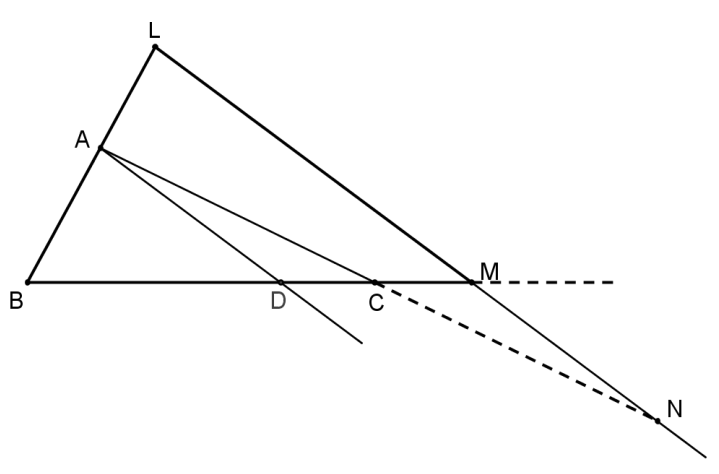

Figura 2: $\overleftrightarrow{A D} \| \overleftrightarrow{L M}$

Aplicando o Teorema de Tales às paralelas $\overleftrightarrow{A D}$ e $\overleftrightarrow{L M}$ que cortam, também, as secantes $\overleftrightarrow{A N}$ e $\overleftrightarrow{D M}$ em partes proporcionais. Assim

$$
\frac{M D}{N A}=\frac{M C}{N C} \Longrightarrow \frac{M D}{N A} \cdot \frac{N C}{M C}=1
$$

Multiplicando (1) e (2), temos que

$$
\frac{L A}{L B} \cdot \frac{M B}{M C} \cdot \frac{N C}{N A}=1
$$

Reciprocamente, sejam $L, M$ e $N$ pontos pertencentes as retas suportes $\overleftrightarrow{A B}, \overleftrightarrow{B C}$ e $\overleftrightarrow{C A}$, respectivamente, de um $\triangle A B C$ tais que satisfazem a relação (3). Seja $N^{\prime}$ o ponto de intersecção de $\overleftrightarrow{L M}$ com $\overleftrightarrow{A C}$, conforme figura abaixo.

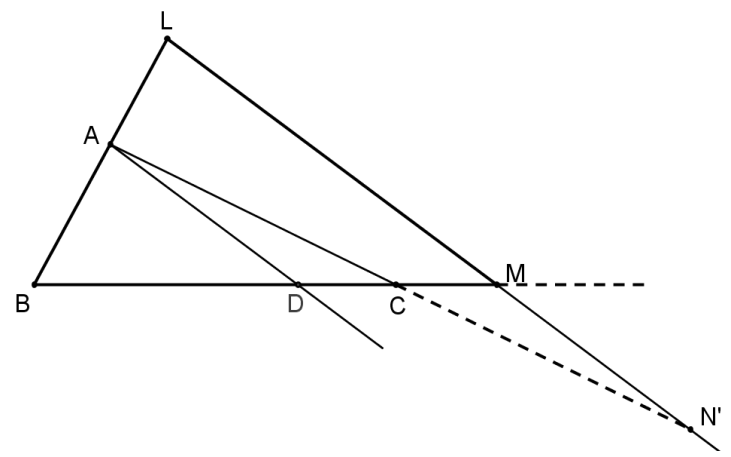

Figura 3: $\overleftrightarrow{L M} \cap \overleftrightarrow{A C}=\left\{N^{\prime}\right\}$

Pelo que foi provado, temos que

$$
\frac{L A}{L B} \cdot \frac{M B}{M C} \cdot \frac{N^{\prime} C}{N^{\prime} A}=1
$$

De (3) e (4), temos $\frac{N C}{N A}=\frac{N^{\prime} C}{N^{\prime} A}$. Como existe apenas um único ponto que divide o segmento $\overline{C A}$ numa dada razão, temos que $N^{\prime}=N$.

Portanto $L, M$ e $N$ são colineares.
Observação 2.1. Quando utilizamos medidas geométricas dos segmentos envolvidos, o Teorema de Menelaus é válido parcialmente, pois a recíproca deste é falsa. Por isso, consideramos apenas as medidas algébricas dos segmentos. Como contra-exemplo consideremos, agora, $L, M$ e $N$ como sendo os pontos médios dos lados $\overline{A B}, \overline{B C}$ e $\overline{A C}$ respectivamente, de um $\triangle A B C$. Consideremos as medidas geométricas dos segmentos $\overline{B M}, \overline{C M}, \overline{C N}, \overline{A N}, \overline{A L}$ e $\overline{B L}$. Temos que $\frac{L A}{L B}$. $\frac{M B}{M C} \cdot \frac{N C}{N A}=1$, mas $L, M$ e $N$ não são colineares. Sendo assim, não vale a recíproca do Teorema de Menelaus.

O Teorema de Menelaus também pode ser apresentado como

$$
\frac{\operatorname{sen}(L \widehat{C} A)}{\operatorname{sen}(L \widehat{C} B)} \cdot \frac{\operatorname{sen}(M \widehat{A} B)}{\operatorname{sen}(M \widehat{A} C)} \cdot \frac{\operatorname{sen}(N \widehat{B} C)}{\operatorname{sen}(N \widehat{B} A)}=1
$$

chamada forma Trigonométrica do Teorema de Menelaus.

Teorema 2.2. (Teorema de Ceva) Num triângulo ABC, três cevianas ${ }^{1} \overline{A L}, \overline{B M}$ e $\overline{C N}$ são concorrentes, se e somente se,

$$
\frac{A N}{N B} \cdot \frac{B L}{L C} \cdot \frac{C M}{M A}=1
$$
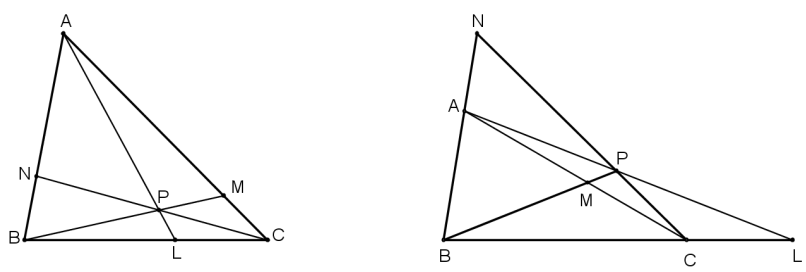

Figura 4: $\overline{A L}, \overline{B M}$ e $\overline{C N}$ são concorrentes em $P$.

Demonstração. Seja $r$ uma reta paralela a $\overline{B C}$ passando por $A$, conforme figura abaixo.

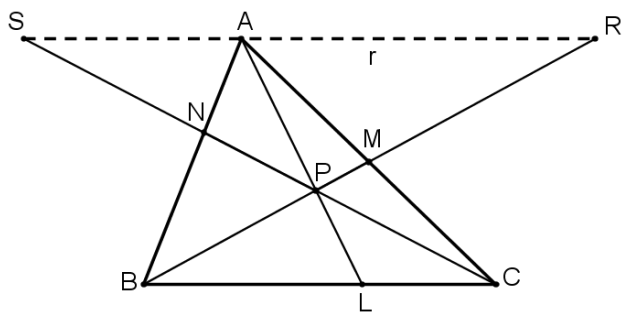

Figura 5: $r \| \overline{B C}$.

Temos as seguintes semelhanças

$$
\begin{gathered}
\triangle A M R \sim \triangle C M B \Longrightarrow \frac{M A}{C M}=\frac{A R}{B C} \\
\triangle B N C \sim \triangle A N S \Longrightarrow \frac{N B}{A N}=\frac{B C}{S A}
\end{gathered}
$$

\footnotetext{
${ }^{1}$ São segmentos que possuem extremidades no vértice e na reta suporte ao lado oposto a este vértice.
} 


$$
\begin{gathered}
\triangle C P L \sim \triangle S P A \Longrightarrow \frac{L C}{S A}=\frac{L P}{A P} \\
\triangle B P L \sim \triangle R P A \Longrightarrow \frac{B L}{A R}=\frac{L P}{A P}
\end{gathered}
$$

De (7) e (8) vem que

$$
\frac{L C}{S A}=\frac{B L}{A R} \text { ou } \frac{L C}{B L}=\frac{S A}{A R}
$$

Multiplicando membro a membro (5), (6) e (9), obtemos

$$
\frac{M A}{C M} \cdot \frac{N B}{A N} \cdot \frac{L C}{B L}=\frac{A R}{B C} \cdot \frac{B C}{S A} \cdot \frac{S A}{A R}=1
$$

ou

$$
\frac{A N}{N B} \cdot \frac{B L}{L C} \cdot \frac{C M}{M A}=1
$$

Seja agora, $\overline{B M} \cap \overline{A L}=\{P\}$ (veja Figura 5) e estendendo o segmento $\overline{P C}$ até intersectar $\overleftrightarrow{A B}$ em $N^{\prime}$. Como $\overline{A L}, \overline{B M}$ e $\overline{C N^{\prime}}$ são concorrentes, aplicando o que foi provado anteriormente temos

$$
\frac{B L}{L C} \cdot \frac{C M}{M A} \cdot \frac{A N^{\prime}}{N^{\prime} B}=1
$$

Mas por hipótese, $\frac{A N}{N B} \cdot \frac{B L}{L C} \cdot \frac{C M}{M A}=1$, o que implica $\frac{A N^{\prime}}{N^{\prime} B}=\frac{A N}{N B}$. Mas isso só ocorre se, e somente se, $N=$ $N^{\prime}$.

Observação 2.2. O Teorema de Ceva também pode ser apresentado como

$$
\frac{\operatorname{sen}(B \widehat{A} L)}{\operatorname{sen}(L \widehat{A} C)} \cdot \frac{\operatorname{sen}(C \widehat{B} M)}{\operatorname{sen}(A \widehat{B} M)} \cdot \frac{\operatorname{sen}(A \widehat{C} N)}{\operatorname{sen}(B \widehat{C} N)}=1
$$

chamada forma Trigonométrica do Teorema de Ceva.

\subsection{Teorema de Ptolomeu e o Teorema de Hiparco}

Neste tópico, apresentamos dois importantes teoremas produzidos no período Alexandrino: O Teorema de Hiparco e o Teorema de Ptolomeu.

Hiparco (180 - 125 a.C.), matemático e astrônomo, nasceu na cidade egípicia de Nicéia. É considerado o fundador da Trigonometria, pois na segunda metade do século II a.C., fez um tratado em doze livros que se ocupa da construção do que deve ter sido a primeira tabela trigonométrica, uma tábua de doze cordas. Evidentemente fez esta tabela para usá-la em sua astronomia.
Os trabalhos de Hiparco e de outros estudiosos da matemática e astronomia, foram reunidos em um compêndio composto de 13 livros pelo matemático e astrônomo Cláudio Ptolomeu (127 - 150 d.C.). No Almagesto, como foi chamado, Ptolomeu desenvolve não somente modelos astronômicos, mas também as ferramentas matemáticas, além da Geometria elementar, necessárias para a Astronomia, entre elas a Trigonometria.

Foi no Almagesto que Ptolomeu apresentou os dois Teoremas que abordaremos a seguir.

Teorema 2.3. (Teorema de Ptolomeu) Num quadrilátero inscritivel, o produto das diagonais é igual à soma dos produtos dos lados opostos.

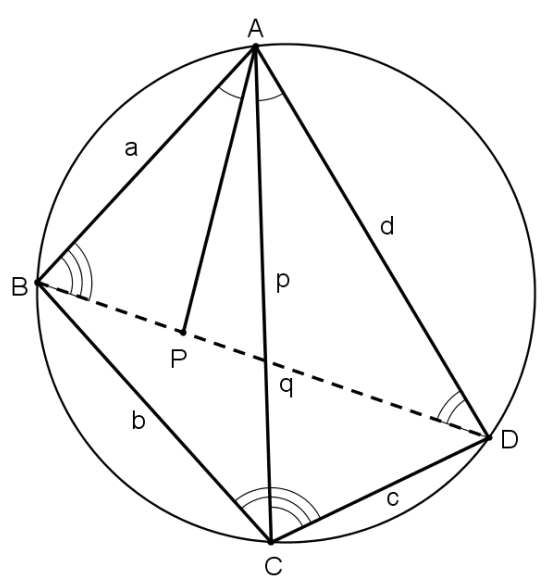

Figura 6: Teorema de Ptolomeu.

Demonstração. Sejam $A B=a, B C=b, C D=c, D A=$ $d, A C=p$ e $B D=q$. Mostraremos que $p \cdot q=a \cdot c+$ $b \cdot d$.

Seja $P$ um ponto sobre $\overline{B D}$ tal que $B \widehat{A P}=C \widehat{A} D$. Como $A \widehat{C} B=P \widehat{D} A$ (ângulo inscrito na circunferência de mesmo arco $\overline{A B}$ ), temos que $\triangle A P D \sim \triangle A B C$, daí

$$
\frac{P D}{b}=\frac{d}{p} \Longrightarrow P D \cdot p=b \cdot d
$$

Analogamente os $\triangle A P B$ e $\triangle A D C$ são semelhantes, o que implica

$$
\frac{B P}{c}=\frac{a}{p} \Longrightarrow B P \cdot p=a \cdot c
$$

Somando (10) e (11) temos

$$
p(\overbrace{P D+B P}^{B D=q})=a \cdot c+b \cdot d \Longrightarrow p \cdot q=a \cdot c+b \cdot d
$$


Teorema 2.4. (Desigualdade de Ptolomeu) Se ABC é um triângulo e $P$ um ponto do plano deste triângulo, então $A B$. $C P+B C \cdot A P \geq A C \cdot B P . A$ igualdade ocorre se, e somente se o quadrilátero $A B C P$ é inscritível.

Demonstração. Sejam $B_{1} \in \overleftrightarrow{A C}, A_{1} \in \overleftrightarrow{B C}$ e $C_{1} \in$ $\overleftrightarrow{A B}$, tais que $\overrightarrow{P C_{1}} \perp \overleftrightarrow{A B}, P B_{1} \perp \overleftrightarrow{A C}$ e $P A_{1} \perp \overleftrightarrow{B C}$. Consideremos os segmentos $\overline{A_{1} C_{1}}, \overline{A_{1} B_{1}}$ e $\overline{B_{1} C_{1}}$. Como $A \widehat{C_{1}} P+A \widehat{B_{1} P}=180^{\circ}$, o quadrilátero $\mathrm{AB}_{1} \mathrm{PC}_{1}$ é inscritível, sendo $\overline{A P}$ um diâmetro do círculo circunscrito (pois $A \widehat{C_{1}} P=90^{\circ}$ ).

Além disso, $B_{1} \widehat{P} C_{1}+C_{1} \widehat{A} B_{1}=180^{\circ}$, mas $C_{1} \widehat{A} B_{1}=$ $180^{\circ}-\widehat{A}$, onde $\widehat{A}$ é um ângulo interno do triângulo $\mathrm{ABC}$. Logo $B_{1} \widehat{P} C_{1}=\widehat{A}$.

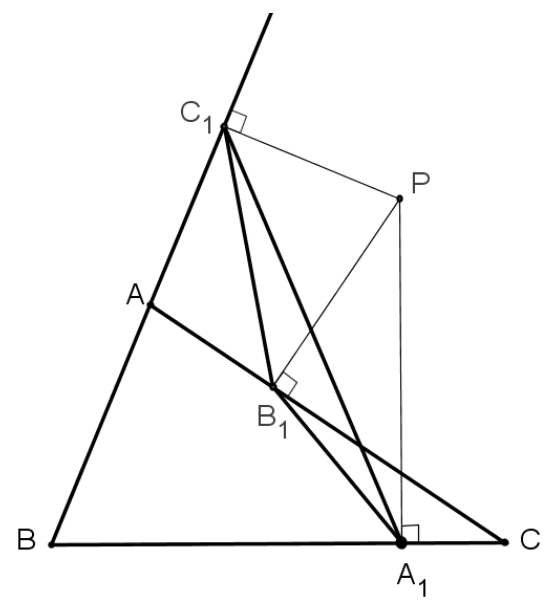

Figura 7: $\mathrm{AB}_{1} \mathrm{PC}_{1}$ é inscritível.

Aplicando a Lei dos Senos no triângulo $\mathrm{PB}_{1} \mathrm{C}_{1}$, temos que

$$
\frac{B_{1} C_{1}}{\operatorname{sen} \widehat{A}}=A P
$$

Pela Lei dos Senos aplicado no triângulo ABC, temos

$$
\operatorname{sen} \widehat{A}=\frac{B C}{2 R}
$$

onde $R$ é o raio do círculo circunscrito ao $\triangle A B C$.

De (12) e (13) vem que

$B_{1} C_{1}=\frac{B C \cdot A P}{2 R}$

Como os quadriláteros $\mathrm{BA}_{1} \mathrm{PC}_{1}$ e $\mathrm{CPB}_{1} \mathrm{~A}_{1}$ também são inscritíveis, temos de modo análogo

$$
A_{1} C_{1}=\frac{A C \cdot B P}{2 R} \text { e } A_{1} B_{1}=\frac{A B \cdot C P}{2 R}
$$

Pela Desigualdade Triangular, temos no triângulo $\mathrm{A}_{1} \mathrm{~B}_{1} \mathrm{C}_{1}$ que

$$
\begin{aligned}
& A_{1} B_{1}+B_{1} C_{1}>A_{1} C_{1} \Longrightarrow \frac{A B \cdot C P}{2 R}+\frac{B C \cdot A P}{2 R}> \\
& \frac{A C \cdot B P}{2 R}
\end{aligned}
$$

Logo

$$
A B \cdot C P+B C \cdot A P>A C \cdot B P
$$

Do Teorema de Ptolomeu e de (14) temos que

$$
A B \cdot C P+B C \cdot A P \geq A C \cdot B P
$$

onde a igualdade ocorre se, e somente se os pontos $A_{1}, B_{1}$ e $C_{1}$ estão alinhados, fazendo com que esta reta seja uma Reta de Simpson-Wallace ${ }^{2}$ e o ponto $P$ pertença ao círculo circunscrito ao triângulo ABC.

Observação 2.3. A Desigualdade de Ptolomeu é um Teorema bem mais geral que o Teorema de Ptolomeu. Esta desigualdade permite descobrir se um quadrilátero $A B C D$ é inscritível ou não, a partir dos valores dos lados e diagonais, sem que seja necessário fazer uma análise dos ângulos. Para isto, basta que se verifique a igualdade $A B \cdot C D+B C \cdot A D=$ $A C \cdot B D$. Se por acaso ocorrer de $A B \cdot C D+B C \cdot A D>$ $A C \cdot B D$, então $A B C D$ não é inscritível.

Teorema 2.5. (Teorema de Hiparco) A razão das diagonais de um quadrilátero inscritível é igual a razão entre as somas dos produtos dos lados que concorrem com as respectivas diagonais.

Demonstração. Considerando o quadrilátero inscritível $\mathrm{ABCD}, \operatorname{com} A B=a, B C=b, C D=c, A D=d$, $A C=p$ e $B D=q$, conforme Figura 8. Mostraremos que $\frac{p}{q}=\frac{a \cdot b+c \cdot d}{a \cdot d+b \cdot c}$.

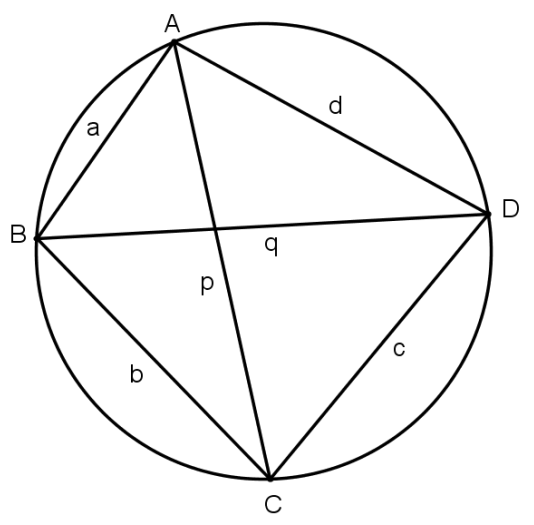

Figura 8: Teorema de Hiparco.

Observemos que a área de $\mathrm{ABCD}$ é equivalente à soma de dois triângulos com um lado comum $\overline{A C}$ ou $\overline{B D}$, o que nos perminte escrever $S_{(A B C)}+S_{(D A C)}=$ $S_{(A B D)}+S_{(C B D)}$.

\footnotetext{
${ }^{2}$ Reta que passa pelos pés das três perpendiculares traçadas a partir de um ponto $\mathrm{P}$ sobre um círculo cincunscrito a um triângulo.
} 
Como esses quatro triângulos possuem o mesmo círculo circunscrito, temos pelo Teorema da área de um triângulo em função dos seus lados e do raio da circunferência circunscrita que que

$$
\begin{aligned}
& \frac{a b p}{4 R}+\frac{c d p}{4 R}=\frac{a d q}{4 R}+\frac{b c q}{4 R} \Longrightarrow \\
& p(a \cdot b+c \cdot d)=q(a \cdot d+b \cdot c)
\end{aligned}
$$

\subsection{Teorema de Feuerbach}

Iniciemos este tópico falando, brevemente, de Leonhard Euler, matemático que produziu cerca de 886 trabalhos em diversas áreas da Matemática, tais como Cálculo, Geometria e Grafos. Além disso, também ficou famoso por seus trabalhos em Mecânica, Óptica e Astronomia. Vários resultados na Geometria levam o seu nome; neste artigo apresentaremos dois deles sem demonstrá-lo: a Reta de Euler e a Circunferência dos nove pontos.

Teorema 2.6. (Reta de Euler) Em um triângulo, o ortocentro $(H)$, o baricentro $(G)$ e o circuncentro $(O)$ estão alinhados. Além disso, o baricentro divide o segmento cujas extremidades são o circuncentro e o ortocentro, na razão 1:2.

O próximo teorema atribuído a Euler, conhecido como Circunferência dos nove pontos, é considerada a primeira circunferência famosa depois da era grega. Euler sabia algumas de suas propriedades, mas foi o matemático francês Poncelet que publicou, em 1821, um artigo demonstrando que para todo triângulo é possível encontrar uma circunferência passando pelos pontos médios dos lados, os pés das alturas e os pontos médios dos segmentos que unem os vértices do triângulo ao ortocentro.

Teorema 2.7. (Circunferência dos nove pontos) Seja ABC um triângulo de circuncentro $\mathrm{O}$ e ortocentro $\mathrm{H}$. Então os pontos médios dos lados, os pés das alturas e os pontos médios dos segmentos que ligam $\mathrm{H}$ aos vértices estão em uma circunferência cujo centro é o ponto médio do segmento $\overline{\mathrm{OH}}$ e cujo raio é a metade do raio da circunferência circunscrita ao triângulo $A B C$.

Falamos que Euler tinha provado algumas propriedades da Circunferência dos nove pontos. Na verdade, Euler provou que passa uma mesma circunferência sobre os vértices dos triângulos medial, que é o triângulo formado unindo os pontos médios do triângulo, e órtico de um triângulo ABC. Mais tarde, este trabalho foi redescoberto por Karl Feuerbach, Matemático alemão do século XIX, que acrescentou uma propriedade notável, que induziu muitos autores a chamar a Circunferência dos nove pontos de Circunferência de Feuerbach.

Feuerbach comprovou que a Circunferência dos nove pontos intersecta as quatro circunferências tritangentes

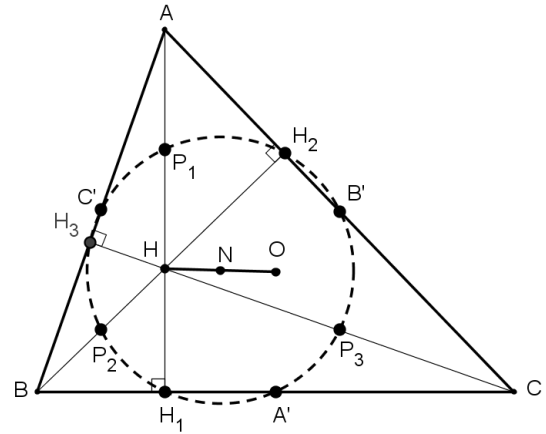

Figura 9: Circunferência dos nove pontos.

de um triângulo $\mathrm{ABC}$ qualquer, ou seja, ela intersecta a circunferência inscrita e às três circunferências exinscrita ${ }^{3}$ ao triângulo $\mathrm{ABC}$.

Para demonstrarmos esse teorema, faremos o uso da Geometria Inversiva. Essa Geometria foi desenvolvida no século XIX por Jacob Steiner e permitiu a solução de problemas que até então estavam sem solução ou com soluções complicadíssimas, se resolvidos pela Geometria Euclidiana. Faremos um breve resumo, mostrando alguns de seus principais resultados.

Definição 2.2. (Conjugados harmônicos) Seja um segmento de reta $\overline{A B}$. Dizemos que dois pontos $M$ e $N$, pertencentes à reta $\overleftrightarrow{A B}$, são conjugados harmônicos de $\overline{A B}$ se

$$
\frac{M A}{M B}=\frac{N A}{N B} .
$$

Como existe apenas um ponto que divide interiormente o segmento $\overline{A B}$ em uma dada razão, concluímos que apenas um deles, $M$ ou $N$, está entre os pontos $A$ e $B$.

Definição 2.3. (Inverso de um ponto) Seja $S=C(O, r)$ um círculo de centro $O$ e raio $r$. Dado um ponto $P$, diferente de $O$, o ponto $P^{\prime}$ na semi-reta $\overrightarrow{O P}$ tal que OP $\cdot O P^{\prime}=r^{2}$ é dito o inverso de $P$ em relação a $S$.

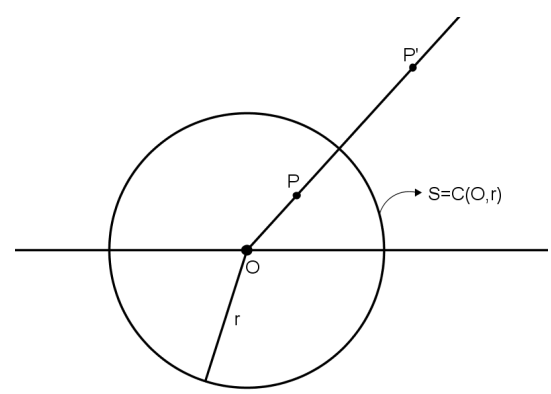

Figura 10: Inverso de um ponto.

\footnotetext{
${ }^{3}$ É uma circunferência externa ao triângulo, tangente a um de seus lados e às extensões dos outros dois.
} 
Nos referimos ao círculo $\mathrm{S}=\mathrm{C}(\mathrm{O}, \mathrm{r})$ como sendo o círculo de inversão e diremos que o ponto $O$ é o centro de inversão.

De uma forma geral, se denotarmos $\mathrm{E}^{2}$ como sendo o plano euclidiano, a transformação $I: E^{2}-\{O\} \rightarrow E^{2}-\{O\}$ que leva qualquer ponto $P \in$ $E^{2}-\{O\}$ a seu inverso referente a $\mathrm{S}=\mathrm{C}(\mathrm{O}, \mathrm{r})$, chama-se inversão. Notemos que I é uma aplicação bijetiva.

Uma vez fixado o círculo de inversão, denotaremos $\mathrm{o}$ inverso de qualquer figura (ou ponto) $\mathrm{X}$ como sendo $X^{\prime}$, ou seja, $\operatorname{Inv}(X)=X^{\prime}$.

Observação 2.4. Considerando a inversão referente a $S=$ $C(O, r)$, temos:

1. Se $P^{\prime}$ é o inverso de $P$, então $P$ e o inverso de $P^{\prime}$.

2. Se $P \neq Q$, então $P^{\prime} \neq Q^{\prime}$.

3. Se $P \in S$, então $P^{\prime}=P$.

Definição 2.4. (Curvas ortogonais) Duas curvas são ortogonais em um ponto de interseção, se as retas tangentes às curvas nesse ponto forem perpendiculares.

Teorema 2.8. (Circunferências ortogonais) Seja $S=C(O, r)$. Se os pontos $P, P^{\prime}$ e O estão alinhados, então $P$ e $P^{\prime}$ são inversos em relação a $S$ se, e somente se, qualquer circunferência que passa por $P$ e $P^{\prime}$ for ortogonal a $S$.

Demonstração. Suponhamos que $P$ e $P^{\prime}$ são pontos inversos em relação à $\mathrm{S}$. Seja $\mathrm{K}=\mathrm{C}(\mathrm{Q}, \mathrm{k})$ uma circunferência com centro em $Q$ e raio $k$, que passa por $P$ e $P^{\prime}$. Como um dos pontos é interno a $S$, as circunferências $S$ e K se intersectam em $G$ e $H$.

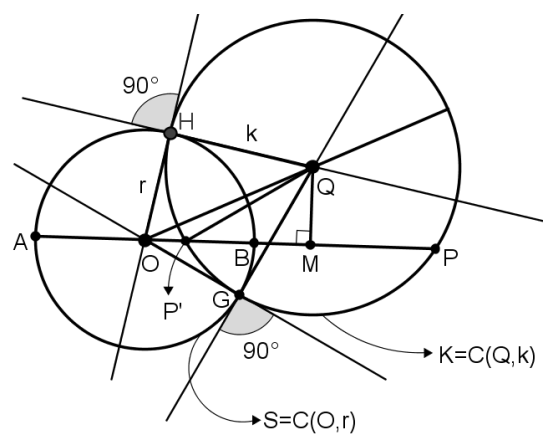

Figura 11: Circunferências ortogonais.

Seja $M$ o pé da perpendicular traçada de $Q$ à reta $\overleftrightarrow{O P}$ conforme figura acima. Do triângulo retângulo OQM vem que

$$
O Q^{2}=O M^{2}+Q M^{2}
$$

Por outro lado, $O M=O P^{\prime}+P^{\prime} M$. Elevando ambos os membros desta equação ao quadrado, temos

$$
\begin{aligned}
O M^{2} & =\left(O P^{\prime}+P^{\prime} M\right)^{2} \\
& =\left(O P^{\prime}+\frac{P P^{\prime}}{2}\right)^{2} \\
& =\left(O P^{\prime}+\frac{O P-O P^{\prime}}{2}\right)^{2} \\
& =\left(\frac{O P+O P^{\prime}}{2}\right)^{2}
\end{aligned}
$$

No triângulo QMP', temos

$$
\begin{aligned}
Q M^{2} & =Q P^{\prime 2}-P^{\prime} M^{2} \\
& =k^{2}-\left(\frac{P P^{\prime}}{2}\right)^{2} \\
& =k^{2}-\left(\frac{O P-O P^{\prime}}{2}\right)^{2}
\end{aligned}
$$

Substituindo as equações (16) e (17) na equação (15) obtemos

$$
O Q^{2}=O P \cdot O P^{\prime}+k^{2}
$$

como por hipótese $O P \cdot O P^{\prime}=r^{2}$, então

$$
O Q^{2}=r^{2}+k^{2}
$$

o que implica que os triângulos OHQ e OGQ são retângulos em $\widehat{H}$ e $\widehat{G}$, respectivamente.

Reciprocamente, seja $\mathrm{K}=\mathrm{C}(\mathrm{Q}, \mathrm{k})$ uma circunferência ortogonal a $\mathrm{S}=\mathrm{C}(\mathrm{O}, \mathrm{r})$ e que passa por $P$ e $P^{\prime}$. Então, se $H$ é um ponto de interseção entre $\mathrm{S}$ e $\mathrm{K}$, a reta $s$ tangente à $\mathrm{K}$ no ponto $H$ é perpendicular à reta $s^{\prime}$ tangente à $\mathrm{S}$ no ponto $H$. Assim, a reta s passa por $O$. Como $O H^{2}=r^{2}$, por Potência de Ponto teremos que

$$
O P \cdot O P^{\prime}=O H^{2}=r^{2}
$$

o que mostra que os pontos $P$ e $P^{\prime}$ são inversos em relação a $S$.

Como consequência direta do teorema acima temos o sequinte corolário:

Corolário 1. Uma circunferência $K=C(Q, k)$ é invariante em relação a uma circunferência de inversão $S=C(O, r)$ se, $e$ somente se, K é ortogonal a $S$.

Teorema 2.9. Os pontos $P$ e $P^{\prime}$ são inversos em relação a $S$ $=C(O, r)$ se, e somente se, $P$ e $P^{\prime}$ são conjugados harmônicos em relação ao diâmetro $\overline{A B}$ determinado pela intersecção da reta $\overleftrightarrow{O P}$ com a circunferência $S$. 


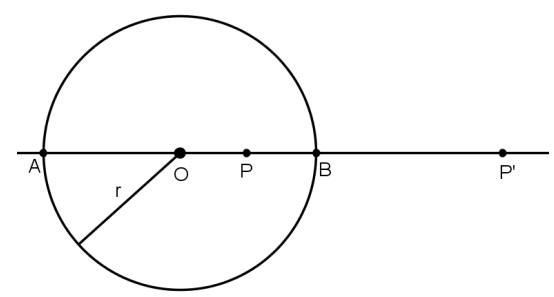

Figura 12: $P$ e $P^{\prime}$ são conjugados harmônicos.

Demonstração. Considerando a Figura 12, temos

$$
\begin{aligned}
& P^{\prime} B=O P^{\prime}-r \\
& P^{\prime} A=O P^{\prime}+r
\end{aligned} \text { e } \begin{aligned}
& P A=r+O P \\
& P B=r-O P
\end{aligned}
$$

então

$$
\frac{P^{\prime} A}{P^{\prime} B}=\frac{O P^{\prime}+r}{O P^{\prime}-r} \text { e } \frac{P A}{P B}=\frac{r+O P}{r-O P}
$$

Assim,

$$
\begin{aligned}
\frac{P^{\prime} A}{P^{\prime} B}=\frac{P A}{P B} & \Leftrightarrow \frac{O P^{\prime}+r}{O P^{\prime}-r}=\frac{r+O P}{r-O P} \\
& \Leftrightarrow \quad\left(O P^{\prime}+r\right) \cdot(r-O P)= \\
\left(O P^{\prime}-r\right) \cdot(O P+r) & \\
& \Leftrightarrow \quad O P \cdot O P^{\prime}=r^{2}
\end{aligned}
$$

Teorema 2.10. (Inversão de reta: Parte I) Seja $S=C(O, R)$. $A$ inversa em relação a $S$ de uma reta s que não passa por $O, e ́$ uma circunferência que passa por $O$.

Demonstração. Seja $A$ o pé da perpendicular traçada de $O$ à reta $s$, e $B$ um ponto qualquer distinto de $A$, pertencente a $s$. Sejam $A^{\prime}$ e $B^{\prime}$ os inversos de $A$ e $B$, respectivamente. Então $O A \cdot O A^{\prime}=O B \cdot O B^{\prime}=r^{2}$, o que implica

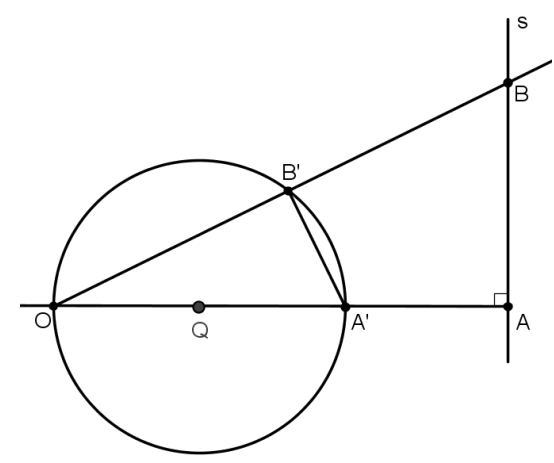

Figura 13: Inversão de reta que não passa por $O$.

$$
\frac{O A}{O B}=\frac{O B^{\prime}}{O A^{\prime}}
$$

e como $B^{\prime} \widehat{O} A^{\prime}=B \widehat{O} A$, concluímos que os triângulos $\mathrm{AOB}$ e $\mathrm{A}^{\prime} \mathrm{OB}^{\prime}$ são semelhantes.

Logo $A^{\prime} \widehat{B}^{\prime} O$ é reto, o que nos mostra que $B^{\prime}$ pertence a uma circunferência $K=(Q, \overline{O Q})$ que tem o segmento $\overline{A^{\prime} O}$ como diâmetro.

Decorre imediatamente que:

Corolário 2. Se s' é uma reta tangente a circunferência $K=$ $(Q, \overline{O Q})$ no ponto $O$, então s'é paralela a s.

Teorema 2.11. (Inversão de reta: Parte II) Seja $S=C(O, R)$. $A$ inversa em relação a $S$ de uma reta s que passa por $O, e ́$ a própria reta s, ou seja, uma reta é invariante quando esta passa pelo centro de inversão.

Demonstração. Se $P \neq O$ é um ponto da reta $s_{\text {, }}$ então, o inverso $P^{\prime}$ de $P$ pertence à semirreta $\overrightarrow{O P}, \operatorname{logo}$, como $P$ e $O$ pertencem à $s$, então, $P^{\prime} \in s$. Portanto $s^{\prime}=\operatorname{Inv}(s)$.

Teorema 2.12. (Inversão de circunferência) Seja $S=C(O, r)$. $A$ inversão em relação a $S$ de uma circunferência $K=C(Q, k)$ que passa por O é uma reta.

Demonstração. Seja $P^{\prime}$ um ponto pertencente ao diâmetro $\overline{O P}$ tal que $O P \cdot O P^{\prime}=r^{2}$.

Consideremos uma reta $s$, que contém $P^{\prime}$ e é perpendicular a $\overline{O P}$, conforme Figura 14 . Seja $B$ um ponto da circunferência $K=(Q, \overline{O Q})$, vamos provar que $B^{\prime}$, pertencente ao segmento $\overline{O B}$, é o inverso de $B$.

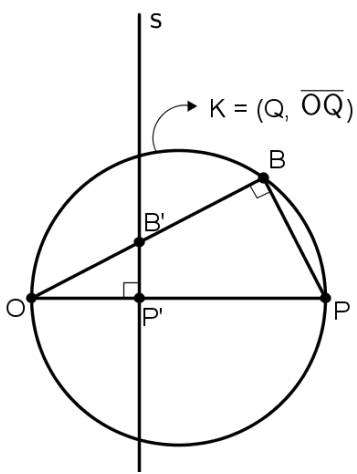

Figura 14: Inversão de circunferência que passa por $O$.

Como os triângulos $\mathrm{OP}^{\prime} \mathrm{B}^{\prime}$ e $\mathrm{OBP}$ são semelhantes, temos que

$$
\frac{O P^{\prime}}{O B}=\frac{O B^{\prime}}{O P} \Longrightarrow O B \cdot O B^{\prime}=O P \cdot O P^{\prime}=r^{2}
$$

O que nos mostra que $B^{\prime}=\operatorname{Inv}(B) . \operatorname{Logo} s=\operatorname{Inv}(K)$ 
Observação 2.5. Considerando $S$ a circunferência de inversão, mostra-se que a inversa de uma circunferência que não passa por O é uma circunferência que, também, não passa por O.

Lema 2.1. Se dois pontos $P$ e $P^{\prime}$ pertencem, respectivamente, às curvas inversas $C e C^{\prime}$, então as tangentes a essas curvas em $P$ e $P^{\prime}$ formam ângulos iguais com a reta $\overleftrightarrow{P P^{\prime}}$.

Teorema 2.13. Se duas curvas $\mathrm{C}_{1}$ e $\mathrm{C}_{2}$ formam um ângulo $\alpha$ em um ponto de interseção $P$, as suas inversas $C_{1}^{\prime}$ e $C_{2}^{\prime}$, na mesma inversão, formarão um ângulo $\alpha$ em um ponto de interseção $P^{\prime}$, inverso de $P$.

Demonstração. Considerando a Figura 15, temos pelo Lema 2.1 que os triângulos $\mathrm{MPP}^{\prime}$ e $\mathrm{NPP}^{\prime}$ são isósceles. Concluímos imediatamente que os ângulos $\alpha$ e $\alpha^{\prime}$ são iguais.

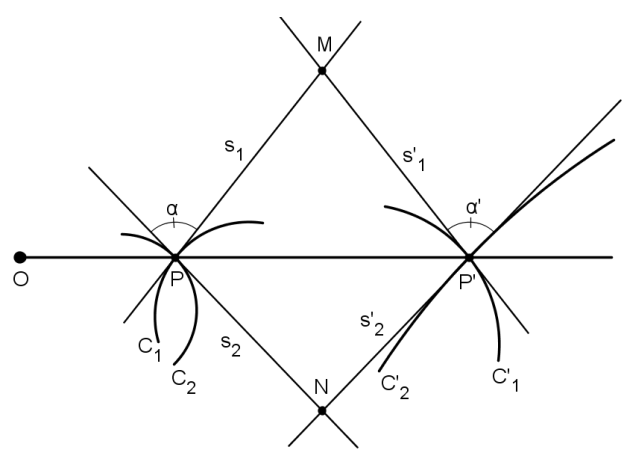

Figura 15: $\alpha=\alpha^{\prime}$.

Enunciamos outro corolário que nos diz que a inversa mantém a tangência entre reta e circunferência, propriedade que será usada na demonstração do Teorema de Feuerbach.

Corolário 3. Seja s uma reta tangente a uma circunferência $K=(Q, k)$ num ponto $P$, então a inversa s' da reta s é tangente a $K^{\prime}$, inversa da circunferência $K$, em $P^{\prime}$.

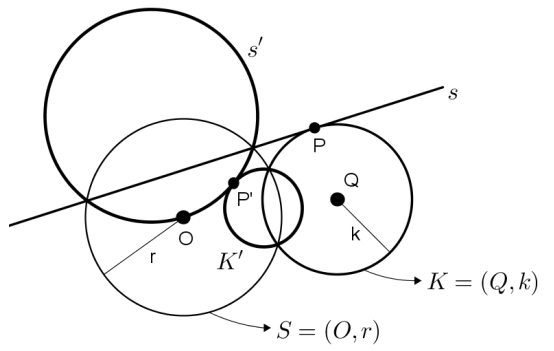

Figura 16: Inversão preserva tangência.

Antes de demonstrarmos o Teorema de Feuerbach vamos enunciar, sem demostrá-los, alguns resultados importantes para a demonstração do teorema supracitado. As demonstrações podem ser vistas em Freitas, 2013.

Lema 2.2. Sejam $M, N$ e $P$ os pontos médio dos lados de um triângulo $A B C, \Omega$ a sua Circunferência dos nove pontos, s uma reta tangente as circunferências inscrita e ex-inscrita a este triângulo e, r uma reta tangente a $\Omega$ por $M$. Então $r$ é paralela a s.

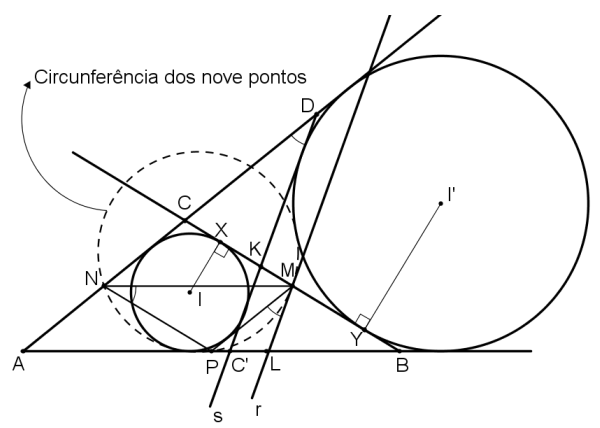

Figura 17: $r \| s$

Lema 2.3. Considerando a Figura 18, M é ponto médio do segmento $\overline{X Y}$.

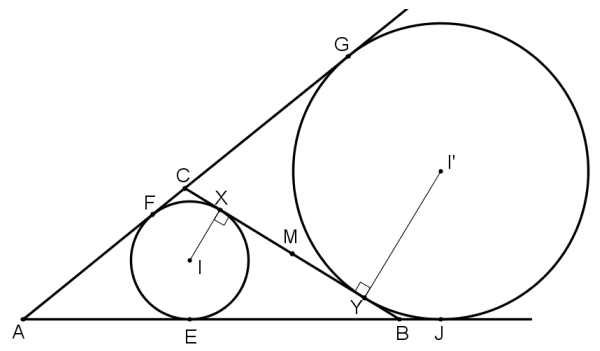

Figura 18: $M$ é ponto médio de $\overline{X Y}$.

Lema 2.4. Considerando a Figura 19, os pontos I e $I^{\prime}$ são conjugados harmônicos referente ao segmentos $\overline{A K}$.

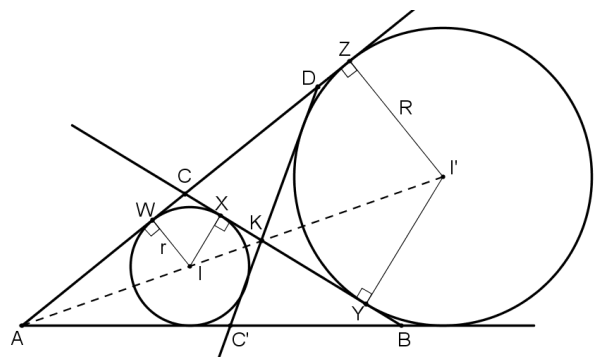

Figura 19: $I$ e $I^{\prime}$ são conjugados harmônicos de $\overline{A K}$.

Lema 2.5. Os pontos $X$ e $Y$, projeções ortogonais de I e $I^{\prime}$, respectivamente, sobre a reta $\overleftrightarrow{B C}$, são conjugados harmônicos em relação ao segmento cuja extremidade são as projeções ortogonais dos pontos $A$ e K. 
Teorema 2.14. (Teorema de Feuerbach) Sejam ABC um triângulo e $\Omega$ sua Circunferência dos nove pontos. Então $\Omega$ é tangente à circunferência inscrita e às três circunferências ex-inscrita ao triângulo $A B C$.

Demonstração. Pelo Lema 2.4, os pontos $I$ e $I^{\prime}$ da Figura 20, são conjugados harmônicos referente ao segmentos $\overline{A K}$. Segue, pelo Lema 2.5, que os pontos $X$ e $Y$ são, também, conjugados harmônicos em relação ao segmentos $\overline{K_{1}}$.

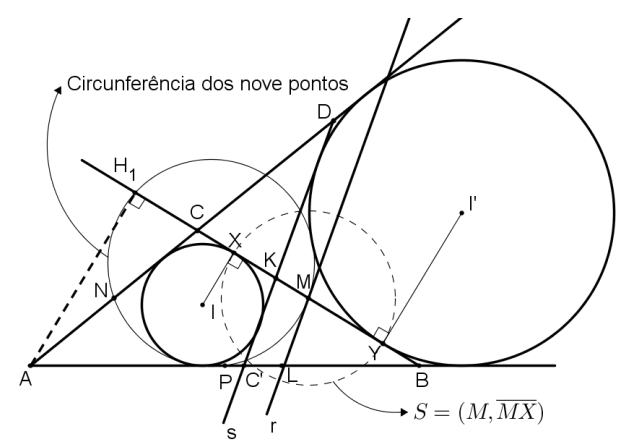

Figura 20: Reta s é a inversa da Circunferência dos nove pontos.

Consideremos, agora, uma circunferência $S=(M, \overline{M X})$, de centro $M$ e que passa por $X$, conforme Figura 20. Pelo Lema 2.3, $S$ também passa pelo ponto $Y$. Como a reta $\overleftrightarrow{B C}$ é tangente as circunferências inscrita e ex-inscrita em $X$ e $Y$, respectivamente, e o diâmetro de $S$ está sobre $\overleftrightarrow{B C}$. Temos que estas circunferências são ortogonais a $S$, donde concluímos, pelo Corolário 1 , que estas circunferências permanecem invariantes em relação $S$. O mesmo acontece com o segmento $\overline{B C}$, pois este segmento passa pelo centro de inversão.

Sendo $\Omega$ a Circunferência dos nove pontos do triângulo ABC. Como $\Omega$ passa pelo centro de inversão, pois passa por $M$, pelo Teorema 2.12, sua inversa é uma reta $s^{\prime}$ que passa pelo ponto $K$, pois $K=\operatorname{Inv}\left(H_{1}\right)$, conforme Teorema 2.9.

Por outro lado, s' é paralela à tangente a $\Omega$ no ponto $M$ (ver Corolário 2). O Lema 2.2 nos mostra que $s^{\prime}$ coincide com $s$, ou seja, $s^{\prime}$ é tangente a circunferência inscrita e ex-inscrita. Logo pelo Corolário $3, \Omega$ é tangente as circunferências inscrita e ex-inscrita.

Por procedimentos similares, mostra-se que $\Omega$ é tangente as outras duas circunferências ex-inscritas.

\section{Algumas Aplicações dos Teoremas Estudados}

Nesta Seção apresentamos algumas aplicações dos teoremas de Menelaus, Ceva e Ptolomeu.

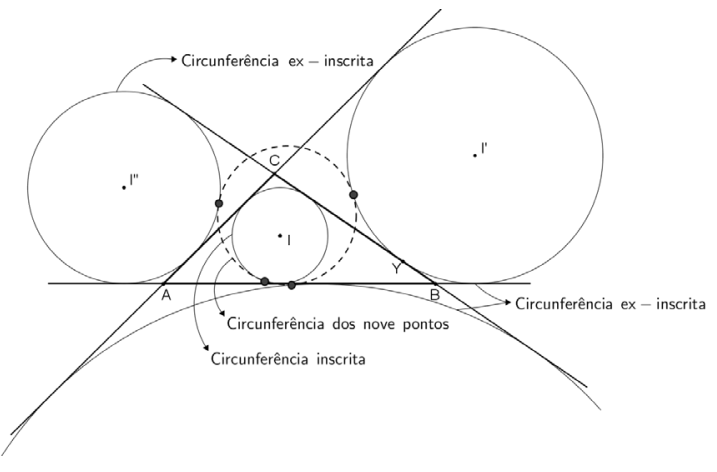

Figura 21: Teorema de Feuerbach.

Exemplo 1. Se dois triângulos situados no mesmo plano estão relacionadas de maneira que as retas que unem vértices homólogos passam por um mesmo ponto (triângulos copolares), então os lados homólogos se cortam nos pontos de uma mesma reta (triângulos colineares). Reciprocamente, triângulos colineares são copolares.

Demonstração. Aplicando o Teorema de Menelaus aos três trios de pontos $\left\{D, R^{\prime}, Q^{\prime}\right\},\left\{E, P^{\prime}, R^{\prime}\right\},\left\{F, Q^{\prime}\right.$, $\left.P^{\prime}\right\}$ em relação aos triângulos $\triangle O Q R, \triangle O R P$, e $\triangle O P Q$, respectivamente, da Figura 22, teremos

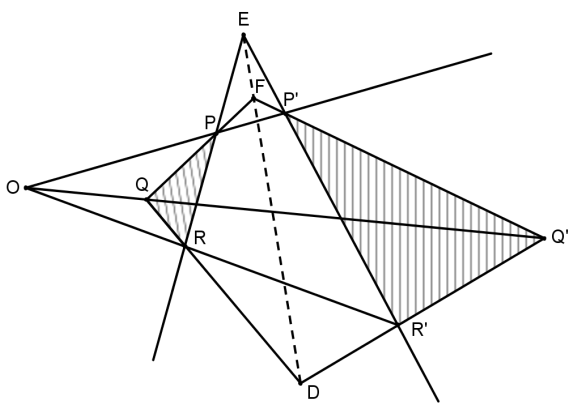

Figura 22: Exemplo 1.

$$
\begin{aligned}
& \frac{Q D}{R D} \cdot \frac{R R^{\prime}}{O R^{\prime}} \cdot \frac{O Q^{\prime}}{Q Q^{\prime}}=1 \\
& \frac{R E}{P E} \cdot \frac{P P^{\prime}}{O P^{\prime}} \cdot \frac{O R^{\prime}}{R R^{\prime}}=1 \\
& \frac{P F}{Q F} \cdot \frac{Q Q^{\prime}}{O Q^{\prime}} \cdot \frac{O P^{\prime}}{P P^{\prime}}=1
\end{aligned}
$$

Multiplicando membro a membro (18), (19) e (20) temos

$$
\frac{Q D}{R D} \cdot \frac{R E}{P E} \cdot \frac{P F}{Q F}=1
$$

Mas a equação (21) nos mostra a recíproca do Teorema de Menelaus aplicado ao $\triangle P Q R$. Portanto $E, F$ e $D$ são colineares. 
Reciprocamente, considerando os $\triangle P Q R$ e $\triangle P^{\prime} Q^{\prime} R^{\prime}$ da Figura 22, temos que os pontos $\overleftrightarrow{P R} \cap \overleftrightarrow{P^{\prime} R^{\prime}}=\{E\}$, $\overleftrightarrow{P Q} \cap \overleftrightarrow{P^{\prime} Q^{\prime}}=\{F\}$ e $\overleftrightarrow{Q R} \cap \overleftrightarrow{Q^{\prime} R^{\prime}}=\{D\}$ são colineares

Sendo $\overleftrightarrow{P P^{\prime}} \cap \overleftrightarrow{R R^{\prime}}=\{O\}$, mostraremos que a reta $\overleftrightarrow{Q Q^{\prime}}$ passa por $O$, ou seja, $\overleftrightarrow{R R^{\prime}} \cap \overleftrightarrow{P P^{\prime}} \cap \overleftrightarrow{Q Q^{\prime}}=\{O\}$

Aplicando aos $\triangle F P P^{\prime}$ e $\triangle D R R^{\prime}$ o que foi mostrado na primeira parte deste teorema, teremos que $\overleftrightarrow{P P^{\prime}} \cap \overleftrightarrow{R R^{\prime}}=\{O\}, \overleftrightarrow{P^{\prime} F} \cap \overleftrightarrow{R^{\prime} D}=\left\{Q^{\prime}\right\}$ e $\overleftrightarrow{F P} \cap \overleftarrow{D R}=\{Q\}$ são colineares e que os dois triângulos possuem os vértices dois a dois sobre três retas concorrentes.

O enunciado do Exemplo 1 é conhecido como Teorema de Gerard Desargues (1591 - 1661). Desarques foi matemático e engenheiro francês, precursor da Geometria Projetiva. Sua principal obra foi sobre as propriedades imutáveis dos círculo, publicada em 1639.

Exemplo 2. (Ponto de Gergonne) Sejam $\overline{A B}, \overline{A C}$ e $\overline{B C}$ os lados de um triângulo $\mathrm{ABC}$, e $\lambda$ um círculo inscrito no triângulo ABC (incírculo). Se $\{X\}=\overline{B C} \cap$ $\lambda,\{Y\}=\overline{A C} \cap \lambda$ e $\{Z\}=\overline{A B} \cap \lambda$, então as cevianas $\overline{A X}, \overline{B Y}$ e $\overline{C Z}$ são concorrentes em um único ponto.

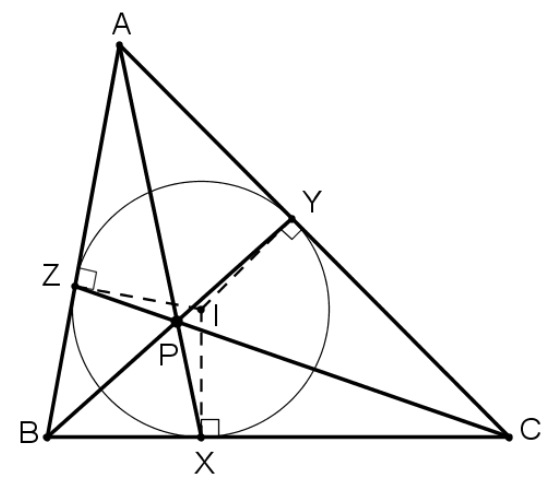

Figura 23: $P$ é o ponto de Gergonne.

Demonstração. Pela Figura 23, temos que $A Z=$ $A Y, B Z=B X$ e $C X=C Y$. Logo

$\frac{B X}{C X} \cdot \frac{A Z}{B Z} \cdot \frac{C Y}{A Y}=\frac{B Z}{C Y} \cdot \frac{A Y}{B Z} \cdot \frac{C Y}{A Y}=1$

Portanto, pelo Teorema de Ceva, as cevianas $\overline{A X}, \overline{B Y}$ e $\overline{C Z}$ são concorrentes em um único ponto.

Exemplo 3. (Ponto de Nagel) Sejam $\overline{A B}, \overline{A C}$ e $\overline{B C}$ os lados de um triângulo $\mathrm{ABC}$, e $\lambda_{1}, \lambda_{2}$ e $\lambda_{3}$ círculos tangentes aos lados do triângulo ABC (ex-círculo). Se $\left\{X_{b}\right\}=\overline{B C} \cap \lambda_{1},\left\{Y_{b}\right\}=\overline{A C} \cap \lambda_{2}$ e $\left\{Z_{b}\right\}=\overline{A B} \cap \lambda_{3}$, então as cevianas $\overline{A X_{b}}, \overline{B Y_{b}}$ e $\overline{C Z_{b}}$ são concorrentes em um único ponto.

Demonstração. Sejam $B C=a, A C=b$ e $A B=c$ e $p$ o semiperímetro de $\mathrm{ABC}$. Temos que $B X_{b}=A Y_{b}=$

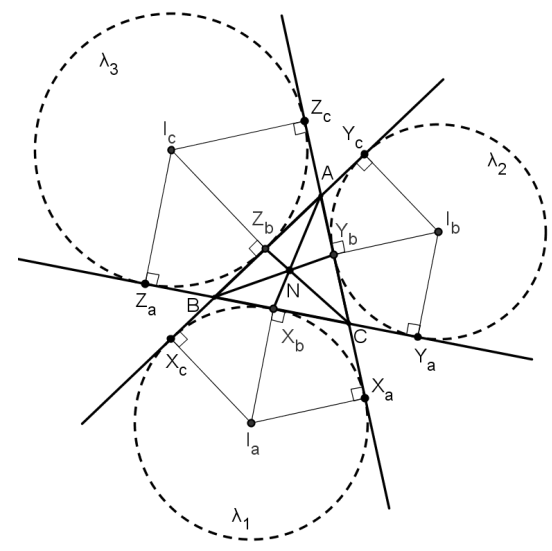

Figura 24: $N$ é o ponto de Nagel.

$p-c$, analogamente temos que $B Z_{b}=C Y_{b}=p-$ $a$ e $A Z_{b}=C X_{b}=p-b$. Logo

$\frac{A Z_{b}}{B Z_{b}} \cdot \frac{B X_{b}}{C X_{b}} \cdot \frac{C Y_{b}}{A Y_{b}}=\frac{p-b}{p-a} \cdot \frac{p-c}{p-b} \cdot \frac{p-a}{p-c}=1$

Portanto, pelo Teorema de Ceva, as cevianas $\overline{A X_{b}}, \overline{B Y_{b}} \mathrm{e} \overline{C Z_{b}}$ são concorrentes em um único ponto.

Exemplo 4. Seja $A_{1} A_{2} A_{3} \cdots A_{n}$ um polígono regular de $\mathrm{n}$ lados. Se $\frac{1}{A_{1} A_{2}}=\frac{1}{A_{1} A_{3}}+\frac{1}{A_{1} A_{4}}$, então este polígono tem 7 lados.

Demonstração. Seja $A_{1} A_{2}=A_{2} A_{3}=\cdots=A_{n-1} A_{n}=$ $a, A_{1} A_{3}=b, A_{1} A_{4}=c$ e $A_{1} A_{5}=d$.

Pela figura abaixo, temos que o quadrilátero $\mathrm{A}_{1} \mathrm{~A}_{3} \mathrm{~A}_{4} \mathrm{~A}_{5}$ é inscritível. Aplicando o Teorema de Ptolomeu temos

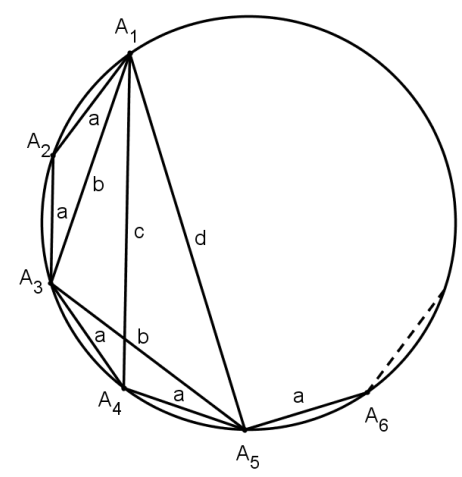

Figura 25: n-ágono regular.

$$
b \cdot c=a \cdot d+a \cdot b \Longrightarrow a \cdot d=b \cdot c-a \cdot b
$$

mas por hipótese

$$
\frac{1}{a}=\frac{1}{b}+\frac{1}{c} \Longrightarrow b \cdot c-a \cdot b=a \cdot c
$$


De (22) e (23) temos

$a \cdot c=a \cdot d \Longrightarrow c=d$

Como $A_{5} A_{6}=a$ e $A_{1} A_{5}=d=c$ devemos ter $A_{1} A_{6}=b$, para isso, basta termos um único ponto sobre a circunferência entre os pontos $A_{1}$ e $A_{6}$. Logo teremos um heptágono regular.

\section{Conclusões}

Neste trabalho procuramos fazer uma abordagem simples de alguns teoremas clássicos da Geometria Plana e torná-los mais conhecidos, pois embora tenham um grande papel na resolução de muitos problemas, estão de certa forma esquecidos tanto no ensino básico quanto no ensino de graduação.

Vimos ao longo do artigo, que muitos desses teoremas clássicos são construídos por meio de teoremas, proposições e corolários bastante conhecidos e ensinados nos diversos níveis de ensino, podendo, portanto, serem abordados, pois não necessitariam de assuntos que não fossem ministrados em tais níveis de ensino. Para o último teorema do Seção 2, fizemos o uso de uma Transformação Geométrica que tem a propriedade de preservar ângulos e que é muito utilizada em problemas que envolvem tangências, a chamada Transformação Inversiva. Transformações Geométricas são aplicações bijetivas de pontos do plano sobre si mesmo, ou seja, uma transformação geométrica transporta um ponto do plano para outro lugar do plano, segundo uma lei de associação. Apesar desse tema não está incorporado às práticas escolares e, até mesmo na formação de professores, sua forma aqui utilizada e suas generalizações para dimensões superiores, são frequentemente utilizadas em demonstrações de problemas geométricos, pois "constituem um campo rico de conexões para raciocinar sobre o plano e o espaço" (BASTOS, 2007).

Acreditamos que tanto o enfoque da realização desse trabalho, com a utilização da Geometria Inversiva, por exemplo, como os teoremas clássicos, que utilizamos simplesmente métodos elementares da Geometria Sintética, pode servir para a melhoria do ensino-aprendizagem de Geometria Euclidiana Plana e possivelmente servir de elemento motivador para alunos e professores que busquem aprimorar seus conhecimentos em Geometria nos seus diversos desdobramentos.

\section{Referências}

BARBOSA, J. (1994). Geometria Euclidiana Plana. Sociedade Brasileira de Matemática.

BASTOS, R. (2007). Notas sobre o ensino de Geometria: Transformações Geométricas. Revista da APM, Lisboa.
CARVAlHO, J. B. P. (1994). Os Elementos de Euclides. RPM-Revista do Professor de Matemática, $\mathrm{n}^{\mathrm{O}} 1$.

COSTA, C., TRALEA, P. (2007). Argumentação e conceito de prova em Matemática. UFF-Universidade Federal Fluminense, Rio de Janeiro.

COXETER, H. S. M., GREITZER, S. L. (1967). Geometry Revisited. The Mathematical Association of America. Washington.

DOLCE, O., POMPEO, J. N. (1985). Fundamentos de Matemática Elementar, $7^{\circ}$ edn. Atual.

FREITAS, V. P. (2013). Algunas Teoremas Clássicos da Geometria Sintética e Aplicações. Universidade Federal do Amazonas.

GUALBERTO, K. J. (2005). Colinearidade e Concorrência na Geometria Plana. Universidade Federal de Santa Catarina.

JOHNSON, R. A. (1960). Advanced Euclidean Geometry. Dover Publications, Inc. New York.

MORGADO, A. C., WAGNER, E., JORGE, M. (2008). Geometria II. VestSeller.

SARMENTO, M. I. (2007). Um Passeio Proveitoso Pelos Círculos de Apolônio. Universidade do Porto, Portugal. 